\title{
El uso de marcadores genéticos en el género Nothofagus con especial referencia a raulí y roble
}

\author{
The use of genetic markers in Nothofagus especially in raulí and roble
}

\author{
Leonardo Ariel Gallo ${ }^{1 *}$, Paula Marchelli, ${ }^{1,2}$, María Marta Azpilicueta ${ }^{1}$, Paula Crego ${ }^{1,2}$ \\ *Autor de correspondencia: ${ }^{1}$ Unidad de Genética Forestal, INTA EEA, Villa Verde s/n, CC 277, 8400 Bariloche, Argentina, \\ Tel.: 542944422731 Fax: 542944424991 lgallo@bariloche.inta.gov.ar \\ ${ }^{2}$ CONICET (Consejo Nacional de Investigaciones Científicas y Técnicas), Argentina
}

\begin{abstract}
SUMMARY
The accurate evaluation of the genetic variation, both for population and evolutionary genetic studies as well as for technological improvement, constitutes a crucial point for proper conclusions and decisions. The use of molecular and biochemical genetic markers in forest tree species has become a key issue for the proper understanding of genetic variation and the resolution of practical problems. Among Nothofagus species, these markers have been used for 20 years. However, in species native to Temperate South American Forests, the first reference dates back to 10 years. This review describes the state of the art of the use of molecular markers among Nothofagus species in general with special concern in Nothofagus nervosa and N. obliqua.
\end{abstract}

Key words: genetic variation, DNA, isozymes, evolutionary processes, population genetics.

\section{RESUMEN}

En trabajos de investigación en genética de poblaciones y evolutiva así como en el desarrollo tecnológico de los programas de mejora, la evaluación correcta de la variación genética es el punto crucial para la conclusión acertada y la toma de decisiones. El uso de marcadores genéticos bioquímicos y moleculares en especies forestales permitió un salto cualitativo en la confiabilidad de la información sobre la variación genética y la resolución en forma contundente de problemas de importancia práctica tanto en genética poblacional como en programas de mejora. En especies del género Nothofagus este tipo de marcadores comenzó a utilizarse hace aproximadamente 20 años, aunque en especies nativas de los bosques templados de Sudamérica la primera referencia data de diez años atrás. El presente trabajo describe los aportes que la genética molecular ha hecho hasta la fecha en los estudios de variación genética del género Nothofagus en general y de Nothofagus nervosa (raulí) y N. obliqua (roble, roble pellín) en particular.

Palabras clave: variación genética, ADN, isoenzimas, procesos evolutivos, genética de poblaciones.

\section{INTRODUCCIÓN}

La información genética, que determina en interacción inseparable con el ambiente el fenotipo observable de los organismos, se encuentra ubicada en diferentes compartimentos celulares (Suzuki et al. 1993), donde se autoduplica, muta y recombina. Si bien en vegetales superiores la mayor parte del ADN se halla en el núcleo, también es posible encontrarlo en organelas como los cloroplastos y las mitocondrias. Moléculas del ARN ribosómico, mensajero y de transferencia son frecuentes además en los ribosomas y en el plasma celular.

En especies sudamericanas del género Nothofagus toda esta información genética ha evolucionado durante aproxi- madamente 100 millones de años (Poole 1987), adaptándose por medio de los fenotipos portadores a enormes cambios geológicos y macroclimáticos que tuvieron lugar en el extremo sur del continente desde el Mesozoico superior. Procesos evolutivos de gran importancia como selección adaptativa, migración y deriva genética, promovidos principalmente durante los procesos de reducción y expansión de las poblaciones, fueron modelando el patrón observable de diferenciación específica adaptada a diversas situaciones de altitud, suelos, humedad, irradiación, etc. En Sudamérica, probable centro de origen evolutivo del género (Swenson et al. 2000), este proceso originó unas nueve especies (Donoso 1987) que cubren gran parte de los aproximadamente 10 millones 
de has de estos bosques templados (Laclau 1997). Algunas de estas especies de Nothofagus ocupan en forma exclusiva nichos bien específicos, mientras que otras comparten hábitats con especies congéneres o en mezclas con coníferas u otras latifoliadas (Donoso y Lara 1995, Laclau 1997).

La conservación de la diversidad genética de estos bosques de Nothofagus y la utilización de sus valiosas especies maderables en programas de domesticación y mejora tendientes a su cultivo, concentran su objetivo en la correcta caracterización y evaluación de la variación genética entre individuos y poblaciones dentro de especies. El raulí (Nothofagus nervosa (Phil.) Dim. et Mil.) y el roble ( $N$. obliqua (Mirb.) Oerst.), especies destacadas por su importancia comercial y por sus particularidades evolutivas, son objeto de estudio desde hace algunos años en trabajos de investigación de los patrones de variación geográfica y genética y de los procesos evolutivos que le dieron origen y los mantienen o modifican (Donoso 1979, Gallo et al. 1997, 2004, 2005, Marchelli y Gallo 1999 y 2004a, Donoso et al. 2004).

En Chile y Argentina se ha comenzado a desarrollar la tecnología necesaria para la domesticación y la mejora genética de ambas especies, contándose en la actualidad con los resultados iniciales de ensayos de campo de procedencias, pruebas de progenie y huertos semilleros (Gallo et al. 2000a, Ipinza y Gutiérrez 2000).

Tanto en los trabajos de investigación en genética de poblaciones y evolutiva cuanto en el desarrollo tecnológico de los programas de mejora, la correcta evaluación de la variación genética es el punto crucial para la acertada conclusión y toma de decisiones. Los primeros estudios de variación geográfica y genética en especies forestales y la evaluación genética de los programas de mejora analizan la distribución de la variación de características morfométricas de importancia adaptativa y/o comercial (Wright 1976). Esta variación continua, asumida como de control genético poligénico, es de difícil caracterización debido a la gran interacción del genotipo con el ambiente. La genética cuantitativa y el análisis estadístico de las fuentes de variación, con numerosos artilugios matemáticos basados en supuestos biológicos poco convincentes y de restringida comprobación, han desarrollado un exagerado marco teórico que apuntala un modelo determinístico y lineal, ampliamente criticado desde hace años (Lewontin 1977, Gallo et al. 1995). Si bien algunas recientes técnicas computacionales de estimación (Gilmour et al. 2000) permitieron mejorar la exactitud de los modelos teóricos cuantitativos más utilizados (Henderson 1975), ha sido principalmente el aporte de la genética molecular lo que ha comenzado a desentrañar recientemente el entendimiento de la conexión entre esas características de importancia adaptativa y/o comercial y su base genética subyacente. El advenimiento de la determinación y uso de marcadores genéticos bioquímicos y moleculares en especies forestales desde hace más de treinta años (Bartels 1971, Bergmann 1971), permitió un salto cualitativo en la confiabilidad de la información sobre la variación genética y la resolución en forma contundente de problemas de importancia práctica tanto en genética poblacional como en programas de mejora.

En especies del género Nothofagus este tipo de marcadores comenzó a utilizarse desde hace poco más de 20 años (Haase 1992). Mientras que en forestales nativas de los bosques templados de Sudamérica la primera referencia de la determinación y uso de marcadores genéticos para estudios de genética poblacional se remonta a diez años atrás (Gallo y Geburek 1994), su utilización en estudios de variación genética de raulí y roble se reporta por primera vez unos años después (Gallo et al. 1997).

La aplicación potencial de algunas técnicas biotecnológicas en el género Nothofagus ha sido descrita en forma teórica por Paredes et al. (2000). Sin llegar a ser exhaustiva, la presente contribución pretende describir los variados aportes que la genética molecular ha hecho hasta la fecha en los estudios de variación genética del género Nothofagus en general y de raulí y roble en particular.

\section{LOS MARCADORES GENÉTICOS}

La unidad evolutiva y la unidad de mejora es la población. Tanto la selección natural como los otros factores evolutivos y la selección artificial, impuesta por el mejorador, actúan directamente sobre las frecuencias génicas poblacionales. Son las proporciones de los genes en las poblaciones las que se ven modificadas permitiendo avanzar en el camino evolutivo de poblaciones naturales o acumular genes de particulares aptitudes adaptativas, de crecimiento, forma, etc., en la población mejorada. Es por ello que la identificación inequívoca de genes y el monitoreo de los cambios de las frecuencias génicas, aun en genes neutros sin relación directa con procesos selectivos, constituyó siempre una aproximación deseada tanto en genética evolutiva cuanto en genética cuantitativa. La herramienta que permitió tal aproximación son los denominados marcadores genéticos. Estos constituyen fenotipos directamente relacionados con la presencia de variantes genéticas, de modo tal que la observación de uno de esos fenotipos permite identificar el marcador genético en cuestión. A pesar de ello, en numerosas técnicas moleculares la relación no es tan directa. Numerosos artefactos metodológicos pueden modificar la expresión fenotípica de una variante genética y de esta manera hacer inestable y poco confiable su interpretación en diferentes análisis de una misma muestra en el mismo o en diferentes laboratorios.

Las variantes genéticas de un locus con dos alelos pueden expresarse fenotípicamente en forma dominante, es decir, una de las variantes homocigotas y el heteroci- 
gota presentan el mismo fenotipo; o en forma codominante, es decir, es posible distinguir fenotípicamente ambas variantes homocigotas de la heterocigota. Con este último tipo de marcadores genéticos es posible y altamente recomendable realizar el análisis genético de la segregación observada en un conjunto de individuos con grado de parentesco conocido. De esta forma, una vez formulada alguna de las hipótesis de segregación mendeliana se ponen a prueba su expresión cuali y cuantitativa, esto último por medio del análisis estadístico con la debida valoración de su significancia.

Cumplido este requisito, es posible denominar a la expresión fenotípica observada "marcador génico", es decir, aquel que establece una relación inequívoca entre ella y la presencia del gen que la controla (Hattemer et al. 1993). El análisis genético de la variación fenotípica es, por lo tanto, la única comprobación aceptable para poder referirse a genes o genotipos y locus o loci génicos. Sin él, sólo se deberá hablar de variantes genéticas o genes putativos. Resulta obvio, entonces, que en estudios de genética poblacional y evaluaciones de genética cuantitativa sea siempre recomendable, aunque no imprescindible, trabajar con marcadores génicos.

Los primeros marcadores génicos fueron morfológicos y corresponden a los determinados por Mendel en sus históricos y notables estudios con material segregante de Pisum sp. y Phaseolus sp., a partir de los cuales formuló las leyes que aún hoy rigen en la genética (Mendel 1866). En especies forestales no son muchos los trabajos en los que se hayan detectado marcadores morfológicos con segregación mendeliana. Uno de los más relevantes, y quizás el primero, corresponda al estudio de la segregación de plantas albinas en la descendencia de individuos de Picea abies (Langner 1953). Otros ejemplos corresponden al control por un gen simple dominante del contenido de antocianina en vacuolas de plántulas de $B e$ tuna sp. (Hattemer et al. 1990) o el contenido relativo de terpenos en especies del género Pinus (Squillace 1977).

Teniendo en cuenta lo mencionado anteriormente, se debe considerar que la gran mayoría de los trabajos de genética poblacional de especies forestales utiliza marcadores genéticos ya que carecen de análisis genéticos, de la segregación fenotípica observada. Algunos autores aducen que cuando el patrón de variación fenotípica observado en las enzimas de una especie es parecido al de otra se puede tener cierto grado de confianza de que se trata de los mismos loci y genes. Sin embargo, estudios relativamente recientes probaron que la homología entre zimogramas, aun de especies taxonómicamente emparentadas, puede conducir a errores de interpretación (Pastorino y Gallo 2001). Por este motivo numerosas afirmaciones sobre genes, procesos genéticos y evolutivos publicados en revistas científicas internacionales deberían ser consideradas intrínsecamente heurísticas (Hattemer 1991).

En especies de vegetales superiores la información genética puede presentarse en condición diploide en los genes situados en el genoma nuclear o haploide en aquellos ubicados en el genoma citoplasmático. Los marcadores genéticos pueden entonces clasificarse también según este criterio. En estudios isoenzimáticos con el debido control genético es posible referirse entonces a las diferencias en la constitución diploide entre dos poblaciones como diferencias en la estructura genotípica, mientras que diferencias en las variantes genéticas del ADN de cloroplastos (cpADN) se indican como diferencias en sus haplotipos.

También pueden clasificarse con variados criterios como el polimorfismo en el número de alelos (variantes genéticas de un mismo locus o sitio genético), la neutralidad o si pertenecen o no a regiones codificantes. Existen en este sentido marcadores genéticos nucleares poco variables (e.g. isoenzimas) y otros hipervariables (e.g. microsatélites o repeticiones de secuencias simples). La mayoría de los marcadores utilizados son neutros a la selección. Por otro lado, en general, corresponden a regiones no codificantes del genoma, a excepción de las isoenzimas y de parte de los fragmentos secuenciados.

Por último, otro criterio puede basarse en la complejidad técnica para desarrollar y utilizar el marcador y en el costo del empleo del mismo. Un resumen de los principales marcadores utilizados en diferentes tipos de estudios en el género Nothofagus y sus características más notables se presentan en el cuadro 1 .

Clasificación taxonómica y filogenética. Los primeros trabajos que utilizaron marcadores moleculares en especies del género Nothofagus se refieren a estudios de clasificación taxonómica y filogenética. Haase (1992) utiliza marcadores genéticos isoenzimáticos (loci putativos) para confirmar la separación taxonómica de $N$. menziesii de otras cuatro especies neocelandesas del género. Los resultados de este primer trabajo, aun con un marcador poco variable como las isoenzimas, establecieron la primera coincidencia clasificatoria en el género entre características fenotípicas del grano de polen (la variable discriminante utilizada hasta esa fecha) y la información genética de las especies.

Analizando el polimorfismo de los fragmentos originados por siete enzimas de restricción con la técnica del southern blot en ADN de cloroplasto, Manos et al. (1993) dieron la primera evidencia molecular sobre la conveniencia de considerar al género Nothofagus como perteneciente a la familia monofilética Nothofagaceae, como había sido propuesto previamente en base a caracteres morfológicos por otros autores (Kuprianova 1962, cit. Manos et al. 1993). Los resultados obtenidos en este primer trabajo ubican a esta familia más cerca de la familia Betulaceae que de Fagaceae. En otro estudio más detallado Martin y Dowd (1993) compararon por primera vez las secuencias nucleotídicas del gen rbcL de ADN de cloroplasto para 23 especies de Nothofagus y tres de Fagus, confirmando la separación de ambos géneros en dos familias diferentes. 
Cuadro 1. Algunos criterios de clasificación de diferentes tipos de marcadores moleculares utilizados hasta la fecha en estudios de genética evolutiva y de poblaciones en especies del género Nothofagus (modificado y adaptado de: de Vienne y Santoni 1998).

Some classification criteria for different molecular markers used in population and evolutionary genetics studies in Nothofagus.

\begin{tabular}{|c|c|c|c|c|c|c|c|}
\hline $\begin{array}{l}\text { Técnica } \\
\text { utilizada }\end{array}$ & $\begin{array}{c}\text { ADN } \\
\text { codificante }\end{array}$ & Número & $\begin{array}{l}\text { Codomi- } \\
\text { nancia }\end{array}$ & $\begin{array}{l}\text { Polimor- } \\
\text { fismo }\end{array}$ & $\begin{array}{l}\text { Complejidad } \\
\text { técnica }\end{array}$ & $\begin{array}{l}\text { Tema abordado } \\
\text { en Nothofagus }\end{array}$ & $\begin{array}{l}\text { Referencia bibliográfica } \\
\text { en Nothofagus }\end{array}$ \\
\hline Isoenzimas & $\mathrm{Si}^{\prime}(1)$ & $<30$ & Sí & Bajo & Baja & $\begin{array}{l}\text { Determinación } \\
\text { marcadores, } \\
\text { diversidad y } \\
\text { diferenciación } \\
\text { genéticas, } \\
\text { hibridación, } \\
\text { filogenia, flujo } \\
\text { génico, } \\
\text { conservación. }\end{array}$ & $\begin{array}{l}\text { Haase 1992, 1993ab y } \\
\text { 1994; Gallo 1995; } \\
\text { Premoli 1996, 1997, } \\
1998 \text { y 2003; } \\
\text { Marchelli y Gallo } \\
\text { 2000ab, 2001; Pineda } \\
\text { 2000ab; Azpilicueta y } \\
\text { Gallo 2001 y 2002; } \\
\text { Crego y Gallo 2002 } \\
\text { ab; Carrasco y Eaton } \\
\text { 2002; Gallo 2002 y } \\
\text { 2004; Gallo et al. } \\
\text { 1997, 2000b y 2003; } \\
\text { Marchelli 2002; } \\
\text { Stecconi et al. } 2002 \text { y } \\
\text { 2004; Donoso et al. } \\
\text { 2004; Premoli y } \\
\text { Kitzberger 2005. }\end{array}$ \\
\hline RFLP & No & $\sim$ ilimitado & Sí & Alto & Media & Filogenia. & Manos et al. 1993. \\
\hline $\begin{array}{l}\text { PCR-RFLP } \\
\text { cpADN }\end{array}$ & No & ilimitado & $\mathrm{No}^{(2)}$ & Medio & Media & $\begin{array}{l}\text { Diferenciación, } \\
\text { determinación de } \\
\text { refugios, caminos } \\
\text { migratorios. }\end{array}$ & $\begin{array}{l}\text { Marchelli et al. } 1998 \text {; } \\
\text { Marchelli y Gallo } \\
\text { 2000b, 2004b y 2006; } \\
\text { Azpilicueta et al. } \\
2005\end{array}$ \\
\hline ISSR & No & ilimitado & No & Muy alto & Baja & $\begin{array}{l}\text { Desarrollo, } \\
\text { diversidad y } \\
\text { diferenciación. }\end{array}$ & Mattioni et al. 2002. \\
\hline RAPD & No & $\sim$ ilimitado & No & Muy alto & Baja & $\begin{array}{l}\text { Desarrollo, } \\
\text { diversidad y } \\
\text { diferenciación. }\end{array}$ & Mattioni et al. 2002. \\
\hline $\begin{array}{c}\text { SSR } \\
\text { (Microsat) }\end{array}$ & No & $\begin{array}{l}\text { Algunos } \\
\text { miles }\end{array}$ & Sí & Muy alto & Alta & $\begin{array}{l}\text { Desarrollo, } \\
\text { transferibilidad, } \\
\text { flujo génico. }\end{array}$ & $\begin{array}{l}\text { Marchelli y } \\
\text { Gallo 2000b; } \\
\text { Azpilicueta } \\
\text { et al. 2004; Jones } \\
\text { et al. 2004. }\end{array}$ \\
\hline Secuenciación & Sí/No & ilimitado & No & Muy alto & Alta & Filogenia. & $\begin{array}{l}\text { Martín y Dowd 1993; } \\
\text { Manos 1997; } \\
\text { Manos y Steele 1997; } \\
\text { Setoguchi et al. } 1997 .\end{array}$ \\
\hline
\end{tabular}

(1) En algunos trabajos se les atribuye además valor adaptativo.

(2) ADN haploide.

Un análisis comparativo posterior de secuencias de bases nucleotídicas ubicadas en el gen matK de ADN de cloroplasto reafirmó la ubicación del género Nothofagus fuera de la familia Fagaceae en la familia Nothofagaceae (Manos y Steele 1997). El estudio filogenético de Martin y Dowd (1993) dentro del genéro Nothofagus confirmó la clasificación morfológica de Hill y Read
(1991) y sugiere al subgénero Lophozonia, donde se ubican raulí y roble como los de mayor antigüedad, con una edad de diferenciación de 66 millones de años. Haase (1994) contribuye con otra estimación del tiempo de separación de los subgéneros neocelandeses Fuscospora y Lophozonia en relación a las distancias genéticas estimadas a partir de estudios isoenzimáticos. Un estudio pos- 
terior de Manos (1997) establece una clasificación filogenética dentro de las especies templado-frío del género en base a las secuencias nucleotídicas codificantes y no codificantes de ADN nuclear ribosomal ( $\mathrm{rADN}$ ) y las compara con los resultados previos del análisis de secuencias nucleotídicas de ADN de cloroplasto de Martin y Dowd (1993) y con el árbol filogenético establecido en base a caracteres morfológicos (Hill y Jordan 1993). El árbol consensuado de las tres fuentes de información confirma, entre otras cosas, la ubicación de roble, raulí y $N$. glauca en un mismo clado dentro del subgénero Lophozonia, resultando roble el taxa ancestral del que habrían derivado las otras dos especies que se encuentran más próximas entre sí. Otro estudio comparativo de las secuencias de bases nucleotídicas del espaciador intergénico atpB-rbcL en el ADN de cloroplasto (Setoguchi et al. 1997) confirmó la clasificación de los cuatros subgéneros determinados por Hill y Read (1991) en base a las características polínicas. Estos autores indican que la diversificación del género en los cuatro subgéneros ocurrió antes de completarse la separación de las placas tectónicas de Gondwana durante el Terciario inferior. Recientemente se confirmó que la distribución transoceánica del género Nothofagus es consistente con un evento de vicariancia, mientras que la distribución a través del mar de Tasmania sólo podría ser explicada por procesos de dispersión a larga distancia (Knapp et al. 2005).

Diferenciación y diversidad genéticas. La diferenciación genética entre poblaciones de especies del género Nothofagus junto con consideraciones sobre la probable ubicación de refugios glaciales y los caminos de recolonización posteriores fue analizada por primera vez por Haase (1992) en N. truncata. El mismo autor contribuye posteriormente con otro estudio de diferenciación sobre la probable distancia de polinización y el efecto fundador en N. menziesii (Haase 1993b). En ambos casos utilizó marcadores genéticos isoenzimáticos. En el último trabajo mencionado, considerando las diferencias en las frecuencias de alelos putativos isoenzimáticos entre las poblaciones fragmentadas analizadas, remarca la importancia del "efecto fundador" y de la polinización a gran distancia en la diferenciación de la especie. También mediante el uso de marcadores genéticos isoenzimáticos se analizaron poblaciones de las tres especies de coihues sudamericanos ( $N$. dombeyi, $N$. betuloides y $N$. nitida), encontrándose que las dos primeras especies, de amplia distribución, poseerían una mayor diversidad genética que N. nitida, de distribución más restringida (Premoli 1996, 1997). La autora sugiere que la presencia de cuatro alelos putativos exclusivos en esta última especie estaría indicando su prolongado aislamiento. Por otro lado, la estructura genética a escala local se vio levemente afectada por el modo de regeneración en $N$. dombeyi (Premoli y Kitzberger 2005). En Nothofagus pumilio (lenga), con marcadores genéticos isoenzimáticos (loci putativos), se informa sobre la variación genética intraespecífica, diferenciándose las poblaciones del norte del área de distribución natural de las del sur y sugiriendo algunas hipótesis evolutivas (Premoli 1998) que deberían confirmarse con un mayor número de poblaciones. Recientemente, un trabajo de diferenciación genética altitudinal, a partir del análisis de variación de las frecuencias de alelos putativos, sugeridos mediante análisis isoenzimático, muestra en dos de las tres transectas analizadas una leve tendencia a la disminución de la variación genética con la altura (Premoli 2003). El primer informe sobre el uso de marcadores de tipo "fingerprinting" en coihue, roble y raulí se refiere a la comparación entre marcadores genéticos de ISSR (fragmentos entre repeticiones de secuencias simples) y RAPDs (ADN polimórfico amplificado al azar) (Mattioni et al. 2002). Estos autores encontraron una alta proporción de bandas polimórficas entre los 63 fragmentos generados para ISSR y los 42 para RAPDs con los seis iniciadores utilizados respectivamente. Con estos polimorfismos analizaron la diversidad genética dentro y entre las muestras de las tres especies, encontrando un alto y similar poder discriminante para ambos marcadores que permitió diferenciar claramente a cada una de ellas.

Los primeros estudios de la diversidad y diferenciación genéticas en raulí y roble se realizaron mediante marcadores isoenzimáticos. La técnica de electroforesis de isoenzimas se ajustó, en primer término, para raulí a partir de los protocolos empleados en especies europeas emparentadas del género Fagus. En ambas especies se realizaron análisis genéticos cuali y cuantitativos de la segregación fenotípica observada en los zimogramas, verificándose estadísticamente las hipótesis de segregación propuestas según el método descrito por Gillet y Hattemer (1989). De esta manera se estableció una relación inequívoca entre el fenotipo observado y la presencia del gen que lo controla, determinándose marcadores génicos, algunos de ellos específicos de cada especie (Gallo et al. 1997).

En el caso del raulí se determinaron ocho loci marcadores, identificándose en ellos 28 alelos (Marchelli y Gallo 2000a). Con estos marcadores se analizó la variación genética de 20 poblaciones, cubriendo toda el área de distribución natural de la especie en Argentina con un mínimo de 100 individuos (embriones) por población, determinándose niveles de variación tanto dentro como entre poblaciones, así como las zonas de mayor diversidad genética (Marchelli y Gallo 2000b, 2001a, 2004ab). Los niveles de heterocigosis observada y esperada fueron de $16 \%$ y $18 \%$ respectivamente, en promedio, para el total de las poblaciones analizadas. El número de loci polimórficos por población varió entre $62,5 \%$ y $100 \%$. El número medio de alelos por locus resultó de 3,5. Se observaron también varios alelos raros, es decir, en proporciones menores al 5\%, así como también alelos exclusivos, particularmente entre las poblaciones más occi- 
dentales de la distribución en Argentina, aquellas ubicadas en las cabeceras Oeste de las cuencas lacustres. A pesar de su distribución netamente fragmentada en el lado argentino y a lo largo de cuencas lacustres de origen glacial con orientación Oeste-Este, la variación longitudinal resultó mayor que la latitudinal. La mayor diversidad genética encontrada en las poblaciones del Oeste, probablemente como consecuencia de la discontinuidad de la superficie de la capa de hielo durante el periodo glacial y la posible ocurrencia de refugios, y el mayor porcentaje de hibridación con roble en las del Este habrían contribuido a este patrón de variación.

Tres poblaciones chilenas de raulí analizadas por los mismos autores a modo de comparación mostraron menor diversidad genética que las poblaciones argentinas (Marchelli y Gallo 2004a). Del mismo modo el número medio de alelos por locus de 22 poblaciones chilenas (Pineda 2000a) y 18 poblaciones chilenas (Carrasco y Eaton 2002) resultaron ser levemente menores que el reportado antes para las 20 poblaciones argentinas. Sin embargo, el grado medio de heterocigosis es mayor en las poblaciones chilenas. Esto sería un indicio del carácter fragmentado de la distribución de la especie en Argentina, con restringido flujo génico entre las poblaciones. Si bien esta comparación entre poblaciones a ambos lados de la Cordillera de Los Andes estaría indicando una tendencia, es necesario tener en cuenta que en el caso de las poblaciones chilenas (Pineda 2000a, Carrasco y Eaton 2002) los resultados preliminares obtenidos corresponden a un tamaño muestral sumamente reducido, a alelos putativos y a enzimas, que como en el caso de las peroxidasas son poco estables, presentando variación ambiental y ontogenética.

En roble el análisis de control genético de la variación observada (Gillet y Hattemer 1989) posibilitó la identificación de siete loci marcadores (Azpilicueta y Gallo 2001). Con ellos se analizaron 14 poblaciones argentinas de la especie con un mínimo de 100 individuos (embriones) por población, determinándose un número total de 19 alelos, con valores medios poblacionales de $85 \%$ de loci polimórficos y un número medio de alelos por locus para la especie de 2,71. La diversidad media poblacional observada ( $v$ ) fue de 1,291. La variación genética mostró un patrón de distribución en el que las poblaciones más variables resultaron las del este de la distribución, más cercanas a la zona de ecotono con la estepa. Asimismo, las poblaciones con las mayores frecuencias de alelos específicos de raulí, especie con la que hibrida, se encontraron también al este de las cuencas (Azpilicueta y Gallo 2002). Tal como en el caso de raulí, a pesar de la distribución marcadamente fragmentada de estos bosques, acotada a las cuencas lacustres y con predominancia de vientos del Oeste, no se encontró una fuerte variación latitudinal.

Los niveles de variación encontrados en ambas especies en Argentina resultaron altos, si se tiene en cuenta la reducida área de distribución natural de ambas especies en ese país. Probablemente esto se deba a tres factores principales: a) la ocurrencia de refugios glaciales al este de la Cordillera de Los Andes, tanto en sitios húmedos como en poblaciones marginadas hacia la estepa; b) la gran fragmentación que tuvo lugar con el subsiguiente proceso de aislamiento e inicio de caminos evolutivos diferentes y c) la alta tasa de hibridación natural encontrada entre ambas especies.

Hibridación interespecífica. Existen algunos aportes de la genética molecular al entendimiento del proceso de hibridación entre diferentes especies del género Nothofagus. En general estos estudios han reforzado el conocimiento previo sobre la existencia de hibridación a través de caracteres morfológicos. Tal es el caso de la hibridación entre Nothofagus betuloides y Nothofagus nitida, descritos inicialmente por Donoso y Atienza (1983) a través de caracteres morfológicos y con una confirmación general a través de marcadores isoenzimáticos (Premoli 1996), o el conocido caso de la hibridación entre de Nothofagus pumilio y Nothofagus antarctica que, además de la clara diferenciación morfológica visible en el dimorfismo foliar de los rámulos, ha sido sugerido también con marcadores genéticos isoenzimáticos en función de la mayor heterocigosis encontrada en los supuestos híbridos (Quiroga et al. 2001).

Recientemente, se informó sobre la utilización de ambas aproximaciones, la morfológica y la molecular, para la primera descripción en comunidades naturales de la ocurrencia de hibridación entre una especie de hoja perenne, $N$. dombeyi y otra de hoja caduca $N$. antarctica (Steconni et al. 2002, 2004).

La hibridación natural entre raulí y roble fue descrita por primera vez en poblaciones naturales de estas especies por Donoso et al. (1990) en base a características morfológicas de las hojas, características anatómicas de la madera y análisis de flavonoides. La ocurrencia de la hibridación ya era conocida en arboretos (Stewart 1979, Tuley 1980), proponiéndose posteriormente el nombre de N. x alpina al híbrido resultante (Lennon et al. 1987). A nuevas descripciones morfológicas y de crecimiento realizadas en familias de una prueba de progenie (Gallo 1995) se sumó el uso de marcadores génicos isoenzimáticos para la identificación de híbridos y el monitoreo de la dinámica de hibridación. Se encontraron dos loci génicos (Adh y Pgi) con alelos específicos para cada especie y de segregación independiente (Gallo et al. 1997). Con estos marcadores fue posible diferenciar a ambas especies parentales de los híbridos $F 1$ y de los probables híbridos retrocruzas en muestras de embriones seminales y de yemas y hojas de plántulas, renovales e individuos adultos. Se encontró una alta correlación positiva entre el grado medio de heterocigosis y el crecimiento inicial en altura en las familias con individuos híbridos. Se verificó una alta segregación en los individuos híbridos con res- 
pecto a su crecimiento y fenología en vivero (Crego 1999). Con estos marcadores específicos de especie pudo determinarse además la unidireccionalidad de la hibridación, actuando el raulí principalmente como madre. Se estimó además la tasa de autofecundación y la estrecha relación entre el grado de aislamiento de los árboles de raulí y el porcentaje de híbridos $F 1$ generados (Gallo et al. 1997). Se probó además una significativa variación temporal en la generación de híbridos $F 1$ y retrocruzas analizando para ello, durante dos o tres años consecutivos, la proporción de híbridos generados en los mismos árboles (Crego y Gallo 2002a). El porcentaje de híbridos encontrados en las diferentes familias de una zona de simpatría varió entre 0 y $83 \%$; mientras que en el bosque sólo fue posible estimar un $6 \%$ de individuos adultos híbridos. Observaciones en vivero de daños por heladas tempranas sugirieron un posible mecanismo de selección natural contra los híbridos $F 1$.

En base a todos estos datos y a los inventarios genéticos de la variación genética de ambas especies (Marchelli y Gallo 2001a, Azpilicueta y Gallo 2002) pudo realizarse un análisis de la distribución espacial del fenómeno de hibridación, diferenciando entre híbridos $F 1$ e híbridos retrocruzas. Se constató que diferentes poblaciones se encuentran en diferentes etapas evolutivas en el proceso de hibridación. En un bosque mixto de ambas especies con un grado de simpatría 1:1 se realizó luego un mapeo individual de los árboles adultos y la regeneración de roble y raulí. En esa parcela se llevó a cabo adicionalmente un estudio fenológico de la floración tomando pares de individuos de ambas especies. Se comprobó que los árboles de raulí en las mismas condiciones ambientales florecen antes que los de roble. Debido a ello, se encontró un solapamiento parcial de los momentos de floración, infiriéndose que la nube de polen con posibilidad de fecundar a individuos de la otra especie estaría constituida por una proporción de granos de polen de roble dos veces y media superior a la de raulí. (Crego y Gallo 2002b). Por otro lado, los individuos de raulí ubicados a menor altitud y en simpatría 1:1 con los de roble estarían aislados fenológicamente de los ubicados a mayor altitud en el bosque puro de esta especie.

Un análisis integral de toda esta información permitió realizar una propuesta sobre la dinámica de hibridación entre ambas especies, tanto a nivel local como regional, estableciéndose un modelo conceptual de base experimental para este importante proceso evolutivo (Gallo 2002, 2004). En este modelo se plantea la posibilidad de un equilibrio dinámico en el proceso de hibridación que podría mantenerse indefinidamente y que resulta posible principalmente debido al balance entre las incompatibilidades pre y postcigóticas de los híbridos.

Migración postglacial. Durante las últimas glaciaciones los bosques vieron drásticamente reducida su distribución, sobreviviendo en refugios. Dada la baja tasa de mutación del ADN de cloroplasto y el bajo número de generaciones desde el retiro de los hielos, la presencia de distintos haplotipos entre las poblaciones de una especie se asume como indicadora de un aislamiento prolongado. Por lo tanto, se pueden utilizar estos marcadores para estudiar la historia pre y postglacial de las especies, determinándose la probable localización de refugios glaciales y reconstrucción de las posibles rutas migratorias luego de la retracción de las masas de hielo. La herencia uniparental y la baja tasa de mutación del ADN de cloroplasto permiten su uso en estudios filogeográficos (Kremer y Petit 1993, Petit et al. 1993). De esta manera, se pueden trazar linajes intraespecíficos analizando las poblaciones que comparten un tipo de ADN de cloroplasto (haplotipo).

Un estudio preliminar en poblaciones de raulí en Argentina, utilizando la técnica de PCR-RFLP con primers universales, reveló la existencia de dos haplotipos (determinados por las combinaciones primer/enzima: $\operatorname{trnD}-\operatorname{trnT} /$ TaqI y trnF-trnV/TaqI), uno al norte y otro al sur del volcán Lanín (Marchelli et al. 1998). Estos resultados fueron sorprendentes dada el área de distribución tan reducida en Argentina y llevaron a sugerir la existencia de al menos dos refugios glaciales ubicados en la Cordillera de Los Andes. Posteriormente, un estudio, incluyendo poblaciones de toda el área de distribución, permitió identificar tres nuevos haplotipos. Los patrones de distribución geográfica de los cinco haplotipos encontrados muestran una separación notoria entre la Cordillera de Los Andes y la Cordillera de la Costa. A su vez, dentro de cada cordillera, se observa una variación en sentido latitudinal. Estos resultados sugieren la existencia de refugios adicionales al de la Cordillera de Nahuelbuta (Villagrán 1991), los cuales estarían situados tanto al Este como al Oeste de la Cordillera de Los Andes y sobre ella (Marchelli et al. 2002). La combinación con la información proveniente de los marcadores isoenzimáticos sugiere como posible centro de dispersión para el raulí la zona de las cuencas Lácar-Neltume, donde se detectó la mayor variación intrapoblacional. Por otro lado, la confluencia de dos rutas migratorias en la zona del lago Paimún, en Argentina, habría estado interrumpida por una erupción reciente del volcán Lanín que mantuvo aisladas a dos poblaciones cercanas (Milleron et al. 2005).

Para el estudio iniciado con roble se utilizaron las mismas combinaciones de primers y enzimas de restricción que en el análisis de raulí. Se analizaron 14 poblaciones argentinas distribuidas en cinco cuencas lacustres y 20 poblaciones de distribución chilena, correspondientes a 10 regiones de procedencia de Chile. Se identificaron seis haplotipos en total, todos presentes entre las poblaciones chilenas y dos entre las argentinas. A lo largo de la Cordillera de Los Andes y de la Depresión Intermedia el patrón de distribución de los haplotipos fue similar al encontrado en raulí, ya que se observaron principalmente dos haplotipos con distribución Norte-Sur. 
Adicionalmente se detectaron tres haplotipos en las poblaciones más septentrionales. La Cordillera de la Costa, zona libre de hielo en las últimas glaciaciones, presentó la mayor diversidad con la presencia de los seis haplotipos (Azpilicueta et al. 2005).

Los altos niveles de diferenciación entre poblaciones encontrados para ambas especies indican un alto grado de fijación $\left(G_{S T}\right.$ raulí $=0,931 ; G_{S T}$ roble $\left.=0,888\right)$, lo cual también ha sido encontrado para otras Fagaceae (Petit et al. 2005).

Los resultados obtenidos con los marcadores de ADN de cloroplasto sugieren que estas dos especies han sobrevivido las glaciaciones en múltiples refugios, probablemente ubicados tanto en la Cordillera de la Costa como a ambos lados de la Cordillera de Los Andes. La identificación de haplotipos compartidos indicaría además la coexistencia de ambas especies en algunos refugios comunes y la ocurrencia de hibridación desde hace miles de años.

Flujo polínico. Los marcadores genéticos codominantes, particularmente los hipervariables como las repeticiones de secuencias simples o microsatélites, constituyen una poderosa herramienta para el análisis de parentesco y por ende para el estudio del movimiento polínico. Estos estudios no sólo tienen importancia práctica en la determinación de la contaminación genética de huertos semilleros (Adams 1981), sino especialmente en ayudar a entender la conectividad entre poblaciones fragmentadas de varias especies nativas y para delimitar los alcances geográficos del concepto de población genética. En el primer caso, ese conocimiento encuentra una aplicación inmediata en la definición de medidas de conservación; en el segundo está estrechamente relacionado con la veracidad de las estimaciones de los parámetros cuantitativos utilizados en mejoramiento. Si bien existen numerosos métodos directos e indirectos para estimar el flujo polínico (Hamrick y Nason 2000), recientemente se ha desarrollado uno con ventajas en el muestreo y en la interpretación de los resultados (Smouse et al. 2001).

Un trabajo reciente presenta el desarrollo de marcadores microsatélites nucleares en $N$. cunninghamii (Jones et al. 2004), donde se determinaron 14 loci polimórficos utilizándose un número equivalente de pares de primers de los cuales seis podrían ser utilizados en otras especies del género, entre ellas roble. Paralelamente, con el objeto de determinar este tipo marcadores específicamente para raulí y roble se llevó a cabo en el INRA-UMR BIOGE$\mathrm{CO}$, Francia, un estudio en el que se desarrollaron 15 microsatélites para Nothofagus y se intentó transferir a Nothofagus 34 microsatélites desarrollados para el género emparentado Quercus (Azpilicueta et al. 2004). De los 15 nuevos primers desarrollados, tres resultaron polimórficos tanto en roble como en raulí. La transferencia entre géneros emparentados dio como resultado polimorfismo en cuatro primers desarrollados para Quercus rubra (roble americano) y en un primer desarrollado para Quercus robur (roble europeo). El número de alelos encontrados en el análisis de los ocho SSRs fue de 1-6 por locus, con 3 alelos como el valor más frecuente. El análisis de segregación pudo confirmarse en seis de los ocho primers analizados y en cuatro de estos el genotipo de la madre pudo ser inferido. A partir de estos resultados se cuenta hoy con ocho primers de microsatélites con los cuales llevar a cabo diversos estudios entre los que está previsto un análisis de flujo génico en poblaciones vecinas de roble, así como el monitoreo del flujo génico y de análisis de parentesco en un rodal mixto de raulí y roble. Asimismo se está estudiando con estos marcadores el flujo polínico a escala local en raulí y se comenzará un estudio a escala de paisaje. Estos estudios posibilitarán profundizar en el conocimiento de aquellos procesos dinámicos que modelan la estructura genética de las poblaciones y contar con una herramienta de amplia aplicación en los programas de mejoramiento.

Impacto del manejo silvicultural. Son pocos los trabajos que han intentado relacionar el impacto del manejo silvicultural sobre la estructura genética del bosque (Hosius 1993). En especies del género Nothofagus existe un estudio preliminar realizado con $N$. pumilio bajo diferentes tratamientos silviculturales (floreo, protección y testigo) en Tierra del Fuego, Argentina (Godoy y Gallo 1998). Los marcadores genéticos utilizados en este caso, genotipos y alelos isoenzimáticos, no mostraron diferencias significativas entre los tres tratamientos analizados en el estrato superior del bosque.

A partir del estudio de la hibridación natural interespecífica entre raulí y roble (Gallo et al. 1997) se llegó deductivamente a formular las consecuencias teóricas que habría tenido la corta selectiva realizada en el pasado en la aptitud de las poblaciones mixtas de ambas especies (Gallo 2002, 2004). Raulí ha sido y es aún la especie más valorada $\mathrm{y}$, por lo tanto, la extracción selectiva de esta especie en hábitats compartidos con el roble modificó la estructura específica de esos bosques mixtos. Esto habría traído aparejado el mayor aislamiento de los árboles remanentes de raulí con respecto a otros individuos de su especie y el aumento proporcional de polen de roble en la nube de polen de la población. Ambas modificaciones tenderían a aumentar la generación de individuos híbridos y, debido a la menor aptitud reproductiva de éstos, a disminuir la aptitud reproductiva general de las poblaciones mixtas (Gallo 2004). El manejo silvicultural actual debería, por lo tanto, tratar de compensar esa extracción selectiva del pasado para recomponer la potencialidad evolutiva de ambas especies.

Conservación. Las zonas que concentran los mayores niveles de diversidad son las más importantes desde el punto de vista de la conservación de una especie. A su vez, constituyen el material base tanto para programas de 
mejoramiento como para el cultivo de la especie fuera de su área de distribución.

En el caso de las poblaciones argentinas de raulí, tanto los estudios isoenzimáticos como los de ADN de cloroplasto permitieron distinguir una zona de gran diversidad genética al oeste de los lagos Lácar y Lolog $\left(40^{\circ} 01^{\prime}-40^{\circ} 10^{\prime} \mathrm{S}\right.$ y $\left.71^{\circ} 46^{\prime}-71^{\circ} 35^{\prime} \mathrm{O}\right)$. Esta zona fue sugerida como posible refugio donde la especie sobrevivió durante las glaciaciones y a partir del cual recolonizó el área previamente glaciada (Marchelli y Gallo 2004a). Por su alto valor genético la zona fue declarada de interés por la Administración del Parque Nacional Lanín y se dispuso un pedido de cambio en su categoría de protección de modo que en ella no sea posible la extracción maderera, evitando así la corta de ejemplares de raulí (Gallo et al. 2003). De esta forma este cambio de categoría de protección se constituyó en el primer caso en Argentina y uno de los pocos en el mundo en donde a partir de datos de variación genética se toman medidas concretas en la definición de áreas protegidas.

De la misma manera, con los datos de variación genética de roble se detectaron del lado argentino dos poblaciones de alto valor de conservación por su constitución genética particular que incluye la presencia de alelos exclusivos (Azpilicueta y Gallo 2002). La primera de ellas ubicada en el norte de la distribución natural de la especie en Argentina, precisamente en el comienzo de los bosques andino-patagónicos, en las lagunas de Epulafquén ( $\left.36^{\circ} 49^{\prime} \mathrm{S}, 71^{\circ} 04^{\prime} \mathrm{O}\right)$ se encuentra dentro de un área de protección provincial (provincia del Neuquén). La otra población de particular interés en roble es la de Pilolil (39 $30^{\prime} \mathrm{S}, 70^{\circ} 57^{\prime} \mathrm{O}$ ), que se encuentra en el extremo oriental de la distribución natural de la especie. Los estudios genéticos la identifican como de alta diversidad, con evidencias de antiguos procesos de hibridación natural con raulí y sugiriéndosela como probable refugio glacial de la especie (Azpilicueta y Gallo 2001, 2002).

Mejoramiento genético. Son numerosas y diversas las aplicaciones de los marcadores bioquímicos y moleculares en programas de mejoramiento. Desde la identificación del material (clones, familias, procedencias) hasta el análisis de parentesco, el diseño de los huertos semilleros, la determinación del porcentaje de su contaminación polínica, la verificación de la proporcionalidad de fecundación en un mix de polen, etc. Estos y otros ejemplos han sido descritos en diferentes trabajos desde hace varios años (Adams 1981).

En el caso de raulí, Pineda (2000b) utilizó marcadores genéticos isoezimáticos para comparar la variabilidad genética de un huerto semillero clonal de raulí con la de las poblaciones naturales donde se seleccionaron los individuos clonados. Si bien el número de individuos analizados no fue suficientemente alto como para sacar conclusiones muy contundentes, la variación genética encontrada en el huerto representó con bastante similitud a la de las poblaciones naturales.

Uno de los aspectos iniciales en los programas tradicionales de mejoramiento y forestación es determinar las regiones de procedencia y de transferencia de semillas. En raulí y roble se determinaron regiones de procedencias del lado chileno en base a la variación climática, la distribución geográfica actual, los límites fitogeográficos y la escasa información sobre variación genecológica (Vergara 2000). En Argentina, utilizando los datos de variación genética aloenzimática de raulí (Marchelli y Gallo 2001a y 2004a) y teniendo en cuenta también la variación en ADN de cloroplasto (Marchelli et al. 1998), se propusieron seis zonas de transferencia de semillas (Marchelli y Gallo 2004b) que serán ajustadas con los datos que surjan de los ensayos de orígenes establecidos a campo. Esta información permite establecer medidas de control evitando la contaminación genética dentro de los Parques Nacionales en los que se distribuye la especie y de este modo asistir a la regeneración natural con semillas pertenecientes a una misma zona. Por otro lado, permite concentrar las actividades de selección del programa de mejoramiento dentro de una misma zona de transferencia y con particular énfasis en los centros de mayor diversidad genética.

Otra aplicación de gran importancia en mejoramiento genético surge a partir de la confirmación de variación temporal en el sistema de apareamiento de raulí. Tanto a través del monitoreo de la generación de híbridos $F 1$ (Crego y Gallo 2002b) como en un estudio ad hoc realizado durante dos años seguidos (Marchelli y Gallo 2001b), pudieron verificarse diferencias significativas entre las estructuras genéticas de la descendencia de diferentes años en un mismo árbol o en una misma población. Este resultado debería ser considerado cuando se realizan cosechas de semillas, particularmente teniendo en cuenta la periodicidad en la semillazón (Marchelli y Gallo 1999) y la mejor representatividad genética en las muestras de aquellos años de alta producción de semilla.

\section{CONCLUSIONES}

Existen en la actualidad alrededor de 26 publicaciones científicas que se refieren a diferentes usos de los marcadores bioquímicos y moleculares en especies del género Nothofagus en general, de las cuales poco más de la mitad tratan directa o indirectamente de su aplicación a estudios genéticos en raulí y roble. Adicionalmente, en estas últimas especies esta temática se aborda también en 10 capítulos de libros, tres tesis doctorales, dos trabajos de divulgación y 13 presentaciones a congresos. La principal contribución de la aplicación de esta poderosa herramienta en estas especies se refiere hasta el momento a estudios filogenéticos y de estimación de la variación genética dentro y entre poblaciones. Algunos de los últi- 
mos trabajos comienzan a incursionar más en procesos genéticos de importancia evolutiva, tales como la migración postglacial y la hibridación interespecífica y a desarrollar y emplear marcadores más potentes y nuevos métodos para la mejor comprensión del flujo génico actual y el análisis de parentesco. Las posibilidades de estas herramientas encuentran además un enorme campo de acción en el monitoreo de la modificación de la variación genética como consecuencia del manejo silvicultural y de la mejora genética. El espectro de posibilidades es, por lo tanto, muy amplio y los resultados esperados muy promisorios siempre y cuando se utilicen el marcador, la estrategia de muestreo y el método de análisis estadístico adecuados.

\section{AGRADECIMIENTOS}

Todos los estudios sobre variación genética molecular en raulí y roble se enmarcan en un programa de conservación y utilización de los recursos genéticos de ambas especies, llevado a cabo en estrecha colaboración con el Departamento de Silvicultura, Delegación Técnica Patagonia de la Administración de Parques Nacionales y financiados por diferentes proyectos de investigación (INTA, INTA-APN, IFS, DAAD-GTZ, BID-FONTAR, BID-FONTAGRO, SAGPyA-BIRF, CONICET). A todos ellos nuestro más sincero agradecimiento.

\section{REFERENCIAS}

Adams WT. 1981. Applying isozyme analyses in tree-breeding programs. In Conkle (technical Co-ordinator), Proc. Symp. isozymes of North American Forest Trees and Forest Insects. Berkeley, California, USDA Forest Service Gen. Tech. Rep. PSW-48. p. 60-64.

Azpilicueta MM, LA Gallo. 2001. Análisis de la variación genética en Nothofagus obliqua (Mirb.) Oerst. a través de marcadores génicos isoenzimáticos. In XXX Congreso Argentino de Genética, Mar del Plata, Argentina. Actas. p. 147.

Azpilicueta MM, LA Gallo. 2002. Variación genética alozímica como herramienta de diagnóstico para la conservación e interpretación evolutiva de los bosques de Nothofagus obliqua (Mirb.) Oerst. en Argentina. In Actas XXXI Congreso Argentino de Genética, La Plata. Actas. p. 135.

Azpilicueta MM, H Caron, C Bodénès, LA Gallo. 2004. SSR markers for analysing South American Nothofagus species. Silvae Genetica 53 (5-6): 240-243.

Azpilicueta MM, P Marchelli, LA Gallo. 2005. Common iceage refugia in two southern beeches (Nothofagus spp.). In XXII IUFRO Congress "Forest in the balance: linking tradition and technology", Brisbane, Australia. p. 68.

Bartels H. 1971. Genetic control of multiple esterases from needles and macrogametophytes of Picea abies. Planta (Berl) 99: 283-289.
Bergmann F. 1971. Genetische Untersuchungen bei Picea abies mit Hilfe der Isoenzym-Identifizierung.I. Möglichkeiten für genetische Zertifizierung von Forstsaatgut. Allgemeine Forst- und Jagdzeitung 142: 278-280.

Carrasco B, L Eaton. 2002. Natural history and genetic structure of Raulí (Nothofagus nervosa (Phil.) Dim. et Mil.). Forest Genetics 9 (4): 275-284.

Crego MP. 1999. Variación genética en el comportamiento fenológico y el crecimiento juvenil de progenies puras e híbridas de raulí, Nothofagus nervosa (Phil.) Dim. et Mil. Tesis Lic. en Biología, Centro Regional Universitario Bariloche. Universidad Nacional del Comahue. 105 p.

Crego MP, LA Gallo. 2002a. Local dynamic of interspecific hybridization between two southern beeches (Nothofagus spp.). In DYGEN Conference. "Dynamics and conservation of genetic diversity in forest ecosystems". Strasbourg, France. Abstracts. p. 108.

Crego MP, LA Gallo. 2002b. Variación temporal en la hibridación natural entre Nothofagus nervosa, Raulí y Nothofagus obliqua, Roble Pellín. In XXXI Congreso Argentino de Genética. La Plata. Actas. p. 136.

de Vienne D, S Santoni. 1998. Les principales sources de marqueurs moléculaires. In D. de Vienne, ed. Les marqueurs moléculaires en génétique et biotechnologies végétales. INRA editions. p. 15-17.

Donoso C. 1979. Genecological differentiation in Nothofagus obliqua (Mirb.) Oerst. in Chile. Forest Ecology and Management 2: 53-66.

Donoso C. 1987. Variación natural en especies de Nothofagus en Chile. Bosque 8 (2): 85-97.

Donoso C, J Atienza. 1983. Hibridación natural entre especies de Nothofagus siempreverdes en Chile. Bosque 5 (1): 21-34.

Donoso C, A Lara. 1995. Utilización de los bosques nativos en Chile: pasado, presente y futuro. Capítulo 19. In Armesto J J, C Villagrán, M Kalin Arroyo eds. Ecología de los bosques nativos de Chile. Monografías. Santiago de Chile, Chile, Editorial Universitaria. p. 363-387.

Donoso C, J Morales, M Romero. 1990. Hibridación natural entre roble (Nothofagus obliqua) (Mirb.) Oerst. y raulí (N. alpina) (Poepp. et Endl.) Oerst, en Bosques del sur de Chile. Revista Chilena de Historia Natural 63: 49-60.

Donoso C, LA Gallo, P Donoso, MM Azpilicueta. 2004. Variación en Nothofagus obliqua (Mirb.) Oerst. In Donoso C, A Premoli, LA Gallo, R Ipinza. Variación intraespecífica en las especies arbóreas de los bosques templados de Chile y Argentina. Santiago de Chile, Chile, Editorial Universitaria. p. 79-113.

Gallo LA. 1995. Supuestos híbridos interespecíficos en progenies de Raulí. In XXVI Congreso Argentino de Genética. XXVIII Reunión Anual de la Sociedad Genética de Chile. San Carlos de Bariloche. p. 34.

Gallo LA. 2002. Conceptual and experimental elements to model natural inter-specific hybridisation between two mountain southern beeches (Nothofagus spp). In Degen B, M D Loveless, A Kremer editors. Modelling and experimental research on genetic processes in tropical and temperate forests. Embrapa-Silvolab-Guyane. ISBN 85-87690-140120-131. p. 120-131.

Gallo LA. 2004. Modelo conceptual sobre la hibridación natural interespecífica entre Nothofagus nervosa y N. obliqua. 
In Donoso C, A Premoli, LA Gallo, R Ipinza eds. Variación intraespecífica en las especies arbóreas de los bosques templados de Chile y Argentina. Santiago de Chile, Chile, Editorial Universitaria. p. 397-407.

Gallo LA, C Donoso, P Marchelli, P Donoso. 2004. Variación en Nothofagus nervosa (Phil.) Dim. et Mil. In Donoso C, A Premoli, LA Gallo, R Ipinza. Variación intraespecífica en las especies arbóreas de los bosques templados de Chile y Argentina. Santiago de Chile, Chile, Editorial Universitaria. p. 115-143.

Gallo LA, Th. Geburek, 1994. A short note: Genetics of isozyme variants in Austrocedrus chilensis (Don.) Florin et Boutelje. Phyton 34: 103-107.

Gallo LA, HR Gregorius, HH Hattemer. 1995. Genetic effects on phenotypic traits in Populus inferable from an exploratory analysis of response functions (ARF) in a factorial mating design. Silvae Genetica 44 (5-6): 290-298.

Gallo LA, P Marchelli, A Breitembücher. 1997. Morphologycal and allozymic evidence of natural hybridization between two southern beeches (Nothofagus spp.) and its relation to heterozygosity and height growth. Forest Genetics 4 (1): 13-21.

Gallo L, P Marchelli, P Crego, L Oudkerk, F Izquierdo, A Breitembücher, M González Peñalba, L Chauchard, L Maresca, Cuerpo de Guardaparques P.N. Lanín y Nahuel Huapi, U Mele. 2000a. Variación genética en poblaciones y progenies de Raulí en Argentina. I. Introducción, distribución y variación en características seminales y adaptativas. In Ipinza R, B Gutiérrez y V Emhart eds. Domesticación y Mejora Genética de raulí y roble. Valdivia, Chile. Exsion. p. 133-155.

Gallo LA, Marchelli P, P Crego. 2000b. Variación genética en poblaciones y progenies de Raulí en Argentina. III. Dinámica de hibridación interespecífica entre Raulí y Roble Pellín. In R Ipinza, B Gutiérrez y V Emhart eds. Domesticación y Mejora Genética de raulí y roble. Valdivia, Chile. Exsion. p. 181-196.

Gallo LA, P Marchelli, M González Peñalba, L Chauchard, S Vellido. 2003. Genética de poblaciones en la definición de áreas protegidas. In IV Simposio de Recursos Genéticos para América Latina y el Caribe. Mar del Plata, Argentina. Libro de resúmenes. p. 158.

Gallo LA, P Marchelli, M Pastorino, F Izquierdo, MM Azpilicueta. 2005. Programa de Conservación y Utilización de los Recursos Genéticos. IDIA XXI, Revista de Información sobre investigación y desarrollo agropecuario, Forestales 5 (8): 157-163.

Gillet E, HH Hattemer. 1989. Genetic analysis of isoenzyme phenotypes using single tree progenies. Heredity 63: 135141.

Gilmour A, B Cullis, S Welham, R Thompson. 2000. ASREML Reference Manual. Version Beta. NSW Agriculture. $227 \mathrm{p}$.

Godoy MM, LA Gallo. 1998. Genética y silvicultura en Nothofagus pumilio (lenga). Patagonia Forestal 4 (1): 9-10.

Haase P. 1992. Isozyme variability and biogeography of Nothofagus truncata (Fagaceaea). New Zealand Journal of Botany 30: 315-328.

Haase P. 1993a. Isozyme studies of New Zealand Nothofagus species (southern beech) using leaf extracts. Silvae Genetica 42 (1): 46-51.
Haase P. 1993b. Genetic variation, gene flow, and the "founder effect" in pioneer populations of Nothofagus menziesii (Fagaceae), South Island, New Zealand. Journal of Biogeography 20: 79-85.

Haase P. 1994. Genetic relationships and inferred evolutionary divergence in the New Zealand taxa of Nothofagus- Results from isozyme analysis. Aust. Syst. Bot., 7: 47-55.

Hamrick JL, JD Nason. 2000. Gene flow in forest trees. In Young A, D Boshier, T Boyle eds. Forest conservation genetics. Principles and practice. CSIRO-CABI publishing. p. 81-90.

Hattemer HH. 1991. Genetic analysis and population genetics. In Fineschi S, M E Malvolti, F Cannata, HH Hattemer eds. Biochemical markers in the population genetics of forest trees. SPB Academic Publishing, The Hague, The Netherlands. p. 5-22.

Hattemer HH, W Steiner, D Kownatzki. 1990. Genetic markers in birch. Silvae Genetica 39: 45-50.

Hattemer HH, F Bergman, M Ziehe. 1993. Einführung in die Genetik für Studierende der Forstwissenschaft. J.D. Suerländer's Verlag. Frankfurt am Main. 492 p.

Henderson CR 1975. Best linear unbiased estimator and prediction under a selection model. Biometrics 32: 69.

Hill RS, J Read. 1991. A revised infrageneric classification of Nothofagus (Fagaceae). Botanical Journal of the Linnean Society 105: 37-72.

Hill RS, G J Jordan. 1993. The evolutionary history of Nothofagus (Nothofagaceae). Australian Systematic Botany 6: 111-126.

Hosius B. 1993. Wird die genetische Struktur eines Fichtenbestandes von Durchforstungseingriffen beeinflusst? Forst und Holz. 48 Jahrgang. p. 306-308

Ipinza R, B Gutiérrez. 2000. Estrategia de mejora genética para $N$. alpina y $N$. obliqua en Chile. In Ipinza R, B Gutiérrez, V. Emhart eds. Domesticación y Mejora Genética de raulí y roble. Valdivia, Chile. Exsion. p. 403-418.

Jones R C, R E Vaillancourt, G J Jordan. 2004. Microsatellites for use in Nothofagus cunninghamii (Nothofagaceae) and related species. Molecular Ecology Notes Volume 4 Issue 1 Page 14, doi:10.1046/j.1471-8286.2003.00558.

Knapp M, K Stöckler, D Havell, F Delsuc, F Sebastiani, P Lockhart. 2005. Relax molecular clock provides evidence for long-distance dispersal of Nothofagus (southern beech). PloS Biology 3 (1): 38-43.

Kremer A, JR Petit. 1993. Gene diversity in natural populations of oak species. Ann. Sci. For. 50: 186s-202s.

Kuprianova AL. 1962. Palynological data and the systematics of the Fagales and Urticales. In Soviet reports from the first international palynological conference. Moscow. U.S.S.R Academy of Sciences. p. 17-25.

Laclau P. 1997. Los ecosistemas forestales y el hombre en el sur de Chile y Argentina. Boletín Técnico 34. Fundación Vida Silvestre Argentina. 147 p.

Langner W. 1952/53. Eine Mendelspaltung bei Aurea-Formen von Picea abies (L.) Karst. Als Mittel zur Klärung der Befruchtungsverhältnisse im Walde. Z. Forstgenetik 2: 49-51.

Lennon JA, E S Martin, RA Stevens, DL Wigston. 1987. Nothofagus nervosa (Phil.) Dim. et Mil. The correct name for raulí, Chilean southern beech (N. procera). Arboricultural Journal 11: 323-332. 
Lewontin R. 1976. The relevance of molecular biology to plant and animal breeding. In Proceedings of the International Conference on Quantitative Genetics. E. Pollak, O. Kempthorne and T.B. Bailey Jr. eds.. The Iowa State University Press, Ames. p. 55-62.

Manos PS. 1997. Systematics of Nothofagus (Nothofagaceae) based on rDNA spacer sequences (ITS): taxonomic congruence with morphology and plastid sequences. American Journal of Botany 84 (8): 1137-1155.

Manos PS, KP Steele. 1997. Phylogenetic analyses of "higher" Hamamelididae based on plastid sequence data. American Journal of Botany 84 (10): 1407-1419.

Manos PS, KC Nixon, JJ Doyle. 1993. Cladistic analysis of restriction site variation within the chloroplast DNA Inverted repeat region of selected Hamamelididae. Systematic Botany 18 (4): 551-562.

Marchelli P. 2002. Variabilidad genética en Raulí (Nothofagus nervosa (Phil.) Dim et Mil.), su relación con procesos evolutivos y la importancia en la conservación y utilización de sus recursos genéticos. Tesis Doctoral, Centro Regional Universitario Bariloche, Universidad Nacional del Comahue. 222 p.

Marchelli P, LA Gallo. 1999. Annual and geographic variation in seed traits of Argentinean populations of southern beech Nothofagus nervosa (Phil.) Dim et Mil. Forestry Ecology and Management 121: 239-250.

Marchelli P, LA Gallo. 2000a. Genetic analysis of isozyme variants in open pollinated families of Southern beech Nothofagus nervosa (Phil.) Dim. et Mil. Silvae Genetica 49: 90-98.

Marchelli P, LA Gallo. 2000b. Variación genética en poblaciones y progenies de Raulí en Argentina. II. Variación aloenzimática, de ADN de cloroplasto y de ADN nuclear. In Ipinza R, B Gutiérrez, V Emhart eds. Domesticación y Mejora Genética de raulí y roble. Valdivia, Chile. Exsion. p. 157-179.

Marchelli P, LA Gallo. 2001a. Genetic diversity and differentiation in a southern beech subjected to introgressive hybridisation. Heredity 87: 284-293.

Marchelli P, LA Gallo. 2001b. Variación temporal en el sistema de apareamiento de Nothofagus nervosa. In XXX Congreso Argentino de Genética. Mar del Plata, Argentina. p. 146.

Marchelli P, LA Gallo. 2004a. The combined role of glaciation and hybridization in shaping the distribution of the genetic variation in a Patagonian southern beech. J. of Biogeography 31 (3): 451-460.

Marchelli P, LA Gallo. 2004b. Conservación y utilización de la variación genética en poblaciones argentinas de Raulí (Nothofagus nervosa (Phil.) Dim. et Mil.). IUFRO International Symposium, Valdivia, Chile. Publicación en CD. 17 p.

Marchelli P, LA Gallo. 2006. Multiple ice-age refugia in a Southern Beech of South America as evidenced by chloroplast DNA markers. Conservation Genetics, en prensa, DOI 10-1007/s10592-005-005-9069-6.

Marchelli P, LA Gallo, F Scholz, B Ziegenhagen. 1998. Chloroplast DNA markers reveal a geographical devide across Argentinean southern beech Nothofagus nervosa (Phil.) Dim. et Mil. distribution area. Theoretical and Applied Genetics 97: 642-646.

Marchelli P, MM Azpilicueta, LA Gallo. 2002. Joint analysis of post-glacial migration in two southern beeches
(Nothofagus spp.). In DYGEN Conference. "Dynamics and conservation of genetic diversity in forest ecosystems". Strasbourg, France. Abstracts. p. 157.

Martin P G, JM Dowd. 1993. Using sequences of $r b c \mathrm{~L}$ to study phylogeny and biogeography of Nothofagus species. Aust. Syst. Bot. 6: 441-447.

Mattioni C, M Casasoli, M González, R Ipinza, F Villani. 2002. Comparison of ISSR and RAPD markers to characterize three Chilean Nothofagus species. Theor. Appl. Genet. 104: 1064-1070.

Mendel G. 1866. Versuche über Pflanzen-Hybriden. Verhandlungen des Naturforschenden Vereins in Brünn 4: 3-47.

Milleron M, LA Gallo, P Marchelli. 2005. Caracterización genética de una zona de confluencia de rutas migratorias postglaciales de Raulí (Nothofagus nervosa (Phil.) Dim. et Mil.). In Actas del XXXIV Congreso Argentino de Genética. p. S-164.

Paredes M, V Becerra, LA Gallo, G Moreno. 2000. Aplicaciones potenciales de la biotecnología al estudio de Nothofagus spp. In Ipinza R, B Gutiérrez, V. Emhart eds. Domesticación y Mejora Genética de raulí y roble. Valdivia, Chile. Exsion. p. 419-434.

Pastorino M, LA Gallo. 2001. Linkage relationships as a useful tool to state interspecific gene homology: Case study with isozyme loci in Austrocedrus chilensis (Cupressaceae). Silvae Genetica 50 (5-6): 233-239.

Petit JR, A Kremer, DB Wagner. 1993. Geographic structure of chloroplast DNA polymorphisms in European oaks. Theoretical and Applied Genetics 87: 122-128.

Petit RJ, J Duminil, S Fineschi, A Hampe, D Salvini, GG Vendramin. 2005. Comparative organization of chloroplast, mitochondrial and nuclear diversity in plant populations. Molecular Ecology 14: 689-701.

Pineda AG. 2000a. Variabilidad aloenzimática de $N$. alpina en Chile. In Ipinza R, Gutiérrez B, V Emhart eds. Domesticación y Mejora Genética de raulí y roble. Valdivia, Chile. Exsion. p. 95-119.

Pineda AG. 2000b. Variabilidad isoenzimática del huerto semillero clonal de N. alpina. In Ipinza R, B Gutiérrez, V Emhart eds. Domesticación y Mejora Genética de raulí y roble. Valdivia, Chile. Exsion. p. 297-305.

Poole AL. 1987. Southern Beeches. New Zealand Department of Scientific and Industrial Research. Information Series № 162.148 p.

Premoli AC. 1996. Allozyme polymorphisms, ourcrossing rates, and hybridization of South American. Nothofagus. Genetica 97: 55-64.

Premoli AC. 1997. Genetic variation in a geographically restricted and two widespread species of South American Nothofagus. Journal of Biogeography 24: 883-892.

Premoli A C. 1998. The use of genetic markers to conserve endangered species and to design protected areas of more widespread species. In Proceedings of an International Workshop on Recent Advances in Biotechnology for Tree Conservation and Management (International Foundation for Science, ed.). Santa Catarina, Brazil, Universidade Federal de Santa Catarina. p. 157-171.

Premoli AC. 2003. Isozyme polymorphisms provide evidence of clinal variation with elevation in Nothofagus pumilio. Journal of heredity 94 (3): 218-226. 
Premoli A, T Kitzberger. 2005. Regeneration mode affects spatial genetic structure of Nothofagus dombeyi forests. Molecular Ecology 14: 2319-2329.

Quiroga MP, R Vidal Russell, AC Premoli. 2001. Posible hibridación entre Nothofagus pumilio y N. antarctica: evidencia morfológica e isoenzimática. I. Reunión Binacional de Ecología Argentina-Chile, Bariloche, Argentina. p. 194.

Setoguchi H, J Ono, Y Doi, H Koyama, M Tsuda. 1997. Molecular phylogeny of Nothofagus (Nothofagacea) based on the $a t p B-r b c L$ intergenic spacer of the chloroplast DNA. J. Plant Res. 110: 469-484.

Smouse PE, RJ Dyer, RD Westfall, VL Sork. 2001. Two generation analysis of pollen flow across a landscape. I. Male gamete heterogeneity among females. Evolution 55: 260271.

Squillace AE. 1977. Monoterpene composition of cortical oleoresins in Pinus elliottii and its utility in genetics research. In Seals DT, GI Forrest, JJ Philipson, H Parkes (eds), EEC Symposium on Forest Tree Biochemistry. p. 39-53.

Stecconi M, P Marchelli, J Puntieri, LA Gallo. 2002. Hybridisation between Nothofagus antarctica (deciduous) and $N$. dombeyi (evergreen) (Nothofagaceae) in natural communities. In Southern Connection Bulletin 21: 8-9.
Steconni M, P Marchelli, J Puntieri, P Picca, LA Gallo. 2004. Natural hybridisation between a deciduous (Nothofagus antarctica, Nothofagaceae) and an evergeen ( N. dombeyi) forest tree species as evidenced by morphological and isoenzymatic traits. Annals of Botany 94: 775-786.

Stewart PJ. 1979. Le gener Nothofagus et son utilisation dans la sylviculture Britannique. Rev. For. Fr. 31: 473-482.

Suzuki DT, AJF Grifiths, JH Miller, RC Lewontin. 1993. Introducción al análisis genético. Interamerican. McGrawHill. 800 p.

Swenson U, RS Hill, S Mcloughlin, 2000. Ancestral area analysis of Nothofagus (Nothofagaceae) and its congruence with the fossil record. Australian Systematic Botany 13: 469-478.

Tuley G. 1980. Nothofagus in Britain. Forestry Commis. Forest Record 122. 26 p.

Vergara R. 2000. Regiones de procedencia de N. alpina y $N$. obliqua. In Ipinza R, B Gutiérrez, V. Emhart eds. Domesticación y Mejora Genética de raulí y roble. Valdivia, Chile. Exsion. p. 121-132.

Villagrán C. 1991. Historia de los bosques templados del sur de Chile durante el Tardiglacial y Postglacial. Revista Chilena de Historia Natural 64: 447-460.

Wright JW. 1976. Introduction to Forest Genetics. Academic Press. New York-San Francisco-London. 463 p. 